\title{
Nail Discoloration
}

National Cancer Institute

\section{Source}

National Cancer Institute. Nail Discoloration. NCI Thesaurus. Code C78492.

A change in the color of the nail. 